\title{
An AU-Aperture Lens to Resolve the Vela Pulsar's Radio Emission Region
}

\author{
C.R. Gwinn, M.J. Ojeda, M.C. Britton \\ University of California, Santa Barbara \\ J.E. Reynolds, D.L. Jauncey, E.A. King \\ Australia Telescope National Facility \\ C.S. Flanagan, D.P. Smits \\ Hartebeesthoek Radio Astronomy Observatory
}

R.A. Preston, D.L. Jones

Jet Propulsion Laboratory

\begin{abstract}
We use radio-wave scattering in the Vela supernova remnant, surrounding the Vela pulsar, as an AU-scale lens to study the Vela pulsar's emission region.
\end{abstract}

\section{Introduction}

Diffraction limits of Earth-based instruments are far poorer than the nanoarcsecond resolution required to resolve emission regions of pulsars. However, fluctuations in density of the interstellar plasma can scatter meter- and decimeterwavelength radiation from a pulsar, and so act as a highly corrupt lens. Such interstellar lenses can resolve pulsar's light cylinders. The Vela pulsar is the brightest pulsar at decimeter wavelengths, and is heavily scattered by the Vela supernova remnant, which encloses it. We use that scattering as a lens to study the pulsar.

\section{Interstellar Scattering}

Density fluctuations in the interstellar plasma introduce phase variations across wavefronts from astrophysical sources. The "crinkled" wavefronts propagate onward to the observer, who receives radiation from a range of directions, and with different phases. The phases of the various waves cancel or reinforce to produce a diffraction pattern in the plane of the observer. Because the line of sight moves relative to the scattering material, the diffraction pattern moves across the observer to produce scintillation.

Radio waves from astrophysical sources carry information on structures of those sources. Interferometric observations on different baselines, followed by a 
Fourier transform, can recover this information; so can imaging with a large lens, which accomplishes the same goal by analog means (Clark 1989). The variations in refractive index in the interstellar plasma responsible for radio-wave scattering do not destroy this information on source structure, but add information on the scattering material to it. Indeed, the scattering material acts as an immense, corrupt lens, with aperture equal to that of the region of scattering material from which an observer receives radiation, known as the "scattering disk". Like any optical system, that lens forms a diffraction pattern in the plane of the observer, which is the convolution of the diffraction pattern for a point source (the "pointspread function") with an image of the source (Goodman 1968, 1985; Cornwell et al. 1989). In principle, observations of the diffraction pattern can recover both the distribution of phase variations introduced by the refracting material, and a diffraction-limited image of the source (Cornwell \& Napier 1988).

The diffraction pattern of a point source is fully coherent. Thus, all of the radiation from any point will form interference fringes with that from any other point, if combined by an interferometer in the plane of the observer. However, if the scattering disk resolves the source, then the diffraction pattern need not be spatially coherent. For example, 2 independently-radiating sources will form 2 copies of the diffraction pattern, displaced in the plane of the observer. Radiation from one copy of the diffraction pattern will not form interference fringes with that from the other. However, if the 2 sources radiate coherently - as they will if they are the 2 slits of a Young's interferometer, for example - the 2 copies of the diffraction pattern will form fringes, if combined by an interferometer in the observer plane. If the spatial coherence of the 2 slits is ruined - perhaps by imposing a rapidly-varying random phase in front of one of the slits - the 2 copies will lose their mutual coherence, and the interference fringes in the observer plane will be ruined. In summary, when the spatial coherence of the source changes without changes in source structure, the spatial coherence of the diffraction pattern can change without great change in the intensity of the diffraction pattern.

\section{Observations of the Vela Pulsar}

The Vela pulsar is the brightest pulsar seen from Earth, at wavelengths shorter than $20 \mathrm{~cm}$. It is a young pulsar, still embedded in the remnant of the supernova that formed it (Bailes et al. 1989). Material in the surrounding supernova remnant strongly scatters radiation from the pulsar (Desai et al. 1992). The scattering disk of the Vela pulsar has a diameter of about $2 \mathrm{AU}$, and lies about $100 \mathrm{pc}$ from the pulsar. Acting as a lens, the scattering disk has linear resolution, at the pulsar, of about $1000 \mathrm{~km}$. This easily resolves the pulsar's light cylinder, with diameter $8000 \mathrm{~km}$.

We observed the Vela pulsar with very-long baseline interferometry at $2.3 \mathrm{GHz}$ in October and November 1992. We used the Mark III recording system at antennas at Tidbinbilla, Parkes, and Hobart in Australia; Hartebeesthoek in South Africa; and the 7 antennas of the US Very Long Baseline Array that could observe this southern-hemisphere object for a useful length of time. We correlated the data at the Haystack Observatory using the Mark IIIA correlator. To study any possible changes in structure of the pulsar over the pulse, we gated the sig- 
nal from the pulsar, and formed fringes separately in 3 gates spaced across the pulse.

We find that the form of the diffraction pattern at individual antennas (or on the shortest baselines) remains quite stable over the pulse, after correction for the change in intensity of the pulsar over the pulse profile. Earlier single-dish observations also note this stability (Backer 1975, Cordes et al. 1983). However, correlation between distant antennas appears to decline more rapidly over the pulse. This behavior suggests initially strong spatial coherence ofthe emission region, which declines over the pulse without a change in source size or shape, as discussed in the example above. More quantitative studies of the correlation function, using autocorrelation functions and moments of the distribution function, are in progress.

\section{Discussion}

High spatial coherence is observed in terrestrial lasers and in scattered, pointlike sources. Strongly coherent scattering - or, equivalently, refraction - in the pulsar's magnetosphere could produce the spatial coherence, and its change over a pulse. Pulsar magnetospheres present significant index of refraction to radio waves (Melrose \& Stoneham 1977, Arons \& Barnard 1986, Barnard \& Arons 1986). We suggest that such magnetospheric refraction, of a compact source of radio waves deep within the magnetosphere, could produce spatially-coherent emission extended to some fraction of the light cylinder.

Acknowledgments. We wish to thank R. Capallo for implementing pulsar gating on the Mark IIIA correlator, and the National Science Foundation and National Air and Space Administration for partial support of this work.

\section{References}

Arons, J., \& Barnard, J.J. 1986, ApJ, 302, 120 Backer, D.C. 1975, A\&A, 43, 395

Barnard, J.J., \& Arons, J. 1986, ApJ, 302, 138

Bailes, M., Manchester, R.N., Kesteven, M.J., Norris, R.P., \& Reynolds, J.E. 1989, ApJ, 343, L53

Clark, B.G. 1989, in Synthesis Imaging in Radio Astronomy, ed. R.A. Perley, F.R. Schwab, \& A.H. Bridle, p 1

Cordes, J.M., Weisberg, J.M., \& Boriakoff, V. 1983, ApJ, 268, 370

Cornwell, T.J., \& Napier, P.J. 1988, Radio Sci, 23, 739

Cornwell, T.J., Anantharamaiah, K.R., \& Narayan, R. 1989, JOSA, A 6, 977

Desai, K.M., Gwinn, C.R., Reynolds, J.R., King, E.A., Jauncey, D., Flanagan, C., Nicolson, G., Preston, R.A., \& Jones, D.L. 1992, ApJ, 393, L75

Goodman, J.W. 1968, Introduction to Fourier Optics (New York: McGraw-Hill)

Goodman, J.W. 1985, Statistical Optics (New York: Wiley)

Melrose, D.B. and Stoneham, R.J., 1977, Proc. Astr. Soc. Australia, 3, 120 